\title{
FSR vehicles classification system based on hybrid neural network with different data extraction methods
}

\begin{abstract}
This paper evaluates the performance of Forward Scatter Radar classification system using as so called ñhybrid FSR classification techniquesò based on three different data extraction methods which are manual, Principal Component Analysis (PCA) and z-score. By combining these data extraction methods with neural network, this FSR hybrid classification system should be able to classify vehicles into their category: small, medium and large vehicles. Vehicle signals for four different types of cars were collected for three different frequencies: $64 \mathrm{MHz}, 151 \mathrm{MHz}$ and $434 \mathrm{MHz}$. Data from the vehicle signal is extracted using above mentioned method and feed as the input to Neural Network. The performance of each method is evaluated by calculating the classification accuracy. The results suggest that the combination of z-score and neural network give the best classification performance compares to manual and PCA methods.
\end{abstract}

Keyword: Z-score; Principal component analysis (PCA); Forward scattering radar (FSR); Neural network (NN); Data extraction; Classification 